Recibido: 28/05/2019 --- Aceptado: 04/07/2019 --- Publicado: 15/03/2020

\title{
ESTUDIO BIBLIOMÉTRICO SOBRE REPUTACIÓN DIGITAL Y ECONOMÍA COLABORATIVA (2004-2017)
}

\section{BIBLIOMETRIC STUDY ON DIGITAL REPUTATION AND COLLABORATIVE ECONOMY (2004-2017)}

\begin{abstract}
818 Marián Navarro-Beltrá ${ }^{\mathbf{1}}$ : Universidad Católica San Antonio Murcia. España. mnavarro2@ucam.edu
\end{abstract}

Josep Martínez-Polo²: Universidad Católica San Antonio Murcia. España. mnavarro2@ucam.edu

\section{RESUMEN}

La confianza y la reputación digital son conceptos de vital importancia para el sector del consumo colaborativo, ya que permiten disminuir el riesgo de los intercambios entre desconocidos. Sin embargo, y a pesar de la importancia de este sector económico, parece que las contribuciones intelectuales de la academia de comunicación al discurso sobre la economía colaborativa son insuficientes. Ante esta situación parece conveniente aumentar la literatura académica existente relacionada con el tema de estudio, pero para ello primero es necesario identificar y conocer las investigaciones ya realizadas. Por tanto, con este trabajo se pretende recopilar, revisar y sintetizar la producción científica existente sobre la confianza y la reputación digital en el ámbito del consumo colaborativo y la economía colaborativa. Para ello, se realiza un análisis bibliométrico de los artículos relacionados con el tema de estudio publicados entre 2004 y 2017 en revistas indexadas en Web of Science, Scopus y Dialnet. Este trabajo muestra que el interés académico por esta temática es reciente, que los artículos son de calidad, que están redactados principalmente en inglés y que no existe una revista, un autor, ni un centro de investigación de referencia. Además, se podría afirmar que los artículos publicados en revistas indexadas en JCR se caracterizan por estar redactados en inglés, por tener una mayor colaboración que los no indexados, tanto a nivel de autores como de instituciones, y por contar con más citas en WOS.

PALABRAS CLAVE: Relaciones Públicas - confianza - reputación - consumo colaborativo - economía colaborativa - análisis bibliométrico - reputación digital

\footnotetext{
${ }^{1}$ Marián Navarro-Beltrá: Licenciada en Publicidad y RRPP (2008) por la Universidad de Alicante y Doctora Cum Laude (2013) por la misma universidad. Actualmente es profesora e investigadora en la Universidad Católica de Murcia (UCAM) en el área de Publicidad y RRPP. mnavarro2@ucam.edu

2 Josep Martínez-Polo: Profesor e investigador en la Universidad Católica de Murcia (UCAM) en el área de Publicidad y RRPP. mnavarro2@ucam.edu
} 
Navarro-Beltrá, M. y Martínez-Polo, J.

Estudio bibliométrico sobre reputación digital y economía colaborativa (2004-2017)

\section{ABSTRACT}

Digital Trust and reputation are concepts of vital importance for the collaborative consumption sector, since they allow reducing the risk of exchanges between strangers. However, and despite the importance of this economic sector, it seems to be that the intellectual contributions of the communication academy to discourse on collaborative economy are insufficient is. Given this situation, it seems to be appropriate to increase the existing academic literature related to the subject of study, but for this, it is first necessary to identify and know the research already carried out. Therefore, this paper aims at collecting, reviewing and synthesizing the existing scientific production on digital trust and reputation in the field of collaborative consumption and collaborative economy. For this, a bibliometric analysis of the articles related to the subject of study published between 2004 and 2017 in journals indexed in Webs of Science, Scopus and Dialnet is carried out. This work shows that the academic interest in this subject is recent; that the articles are of quality, that they are written mainly in English and that there is no magazine, author, or reference research center. In addition, it could be said that the articles published in journals indexed in JCR are characterized by being written in English, having a greater collaboration than those not indexed, both at the level of authors and institutions, and by having more citations in WOS.

KEY WORDS: Public Relations - trust - reputation - collaborative consumption sharing economy - bibliometric analysis - digital reputation.

\section{ESTUDO BIBLIOMÊTRICO SOBRE REPUTAÇÃO DIGITAL E ECONÔMIA COLABORATIVA (2004-2017)}

\section{RESUME}

A confiança e a reputação digital são conceitos de vital importância para o setor do consumo colaborativo, já que permitem diminuir o risco dos intercâmbios entre desconhecidos. Entretanto, e apesar da importância da academia de comunicação ao discurso sobre a econômia colaborativa são insuficientes. Diante dessa situação parece conveniente aumentar a literatura acadêmica existente relacionada com o tema de estudo, mas para isso primeiro é necessário identificar e conhecer as investigações já realizadas. Portanto, com este trabalho pretendemos recopilar, revisar e sintetizar a produção cientifica existente sobre a confiança e a reputação digital no âmbito do consumo colaborativo e a economia colaborativa. Para isso, realizamos uma análise bibliométrica dos artigos relacionados com o tema de estudo publicados entre 2004 e 2017 em revistas indexadas na Web of Science, Scopus e Dialnet. Este trabalho mostra que o interesse acadêmico por esta temática é recente, que os artigos são de qualidade, que estão redigidos principalmente em inglês e que não existe uma revista, um autor, nem um centro de investigação de referência. Ademais, pode-se afirmar que os artigos publicados em revistas indexadas em JCR caracterizam-se por estar redigidos em inglês, por ter maior colaboração que os 
Navarro-Beltrá, M. y Martínez-Polo, J.

Estudio bibliométrico sobre reputación digital y economía colaborativa (2004-2017)

indexados, tanto a nível de autores como de instituições, e por contar com mais consultas na WOS.

PALAVRAS CHAVE: Relações Públicas - confiança - reputação - consumo colaborativo - economia colaborativa - análises bibliométrico - reputação digital.

\section{Cómo citar el artículo:}

Navarro-Beltrá, M. y Martínez-Polo, J. (2020). Estudio bibliométrico sobre reputación digital y economía colaborativa (2004-2017). [Bibliometric study on digital reputation and collaborative economy (2004-2017)]. Revista de Comunicación de la SEECI, 51, 83-107. doi: http://doi.org/10.15198/seeci.2020.51.83-107

Recuperado de http://www.seeci.net/revista/index.php/seeci/article/view/596

\section{INTRODUCCIÓN}

Podría considerarse que las relaciones públicas son una disciplina ligada a una actividad profesional que se encuentra en constante evolución (Castillo, 2009, p. 51), ya que está muy vinculada a los avances de la técnica desde la aparición de la imprenta (Grunig y Hunt, 2000, p. 65) y a las nuevas Tecnologías de la Información y la Comunicación (Zapata-Palacios, 2016, p. 105). De esta forma, la existencia de nuevos medios digitales de comunicación y su gestión ha dado lugar al surgimiento de un nuevo paradigma en el sector de las relaciones públicas: las Relaciones Públicas 2.0 (Fuentes, 2018, p. 18). Así, se puede afirmar que en las últimas décadas esta profesión está sufriendo una importante transformación causada por el frecuente uso que la población realiza de las tecnologías basadas en internet (Sellas, 2014, p. 197).

Con el surgimiento de la sociedad red (Castells, 2005, p. 505) y la aparición de internet las personas empiezan a interaccionar y a realizar transacciones con desconocidos, pues en los inicios de este medio en la mayoría de las ocasiones se desconocía la identidad de los usuarios. Ante esta situación, que el consumidor superase la falta de confianza era fundamental para el desarrollo de internet como canal para las relaciones comerciales (Flavián y Guinalíu, 2006, p. 151).

En este contexto, las relaciones públicas adquieren una gran relevancia, ya que ayudan a generar reputación, credibilidad y, sobre todo, confianza (Valdez, Borrayo y Muñoz, 2018, p. 456). En concreto, ganarse la confianza de los usuarios, que es un elemento subjetivo que depende de las referencias recibidas y de la experiencia personal (Sánchez-Alzate y Montoya, 2016, p. 170), era la base para construir una reputación digital positiva (Aced, 2010, p. 84). Circunstancia que resulta de vital importancia- al considerar que la buena reputación del vendedor incrementa la percepción de calidad de sus servicios y productos en las personas que los adquieren a través de internet (Sánchez-Alzate y Montoya, 2017, p. 12). De ahí que las relaciones públicas trabajen para "lograr tan ansiada reputación" (Rivero, 2017, p. 162) y los planes estratégicos relacionados con esta disciplina traten de tener como resultado una reputación favorable (Miyashiro, 2017, pp. 97-98). 
No obstante, el abundante uso de los social media por parte de la sociedad permitió a los stakeholders comunicarse con otros y difundir sus propios mensajes sobre las organizaciones, situación que conlleva la aparición de amenazas para la reputación (Maestro, Cordón y Abuín, 2018, p. 212) al considerar que esta no depende únicamente de lo que las instituciones dicen de sí mismas (gestionado por los departamentos de relaciones públicas y comunicación), pues también depende de lo que los demás dicen de ellas (Aced, 2018, p. 32). En efecto, la penetración de los medios sociales ha conllevado que determinadas personas lancen mensajes que son susceptibles de alcanzar un impacto y una visibilidad que eran impensables en entornos convencionales (Piñeiro-Otero y Martínez-Rolán, 2016, p. 18). Ante este contexto, se puede afirmar que tanto la confianza como la reputación se convierten entonces en elementos fundamentales en el éxito de transacciones a través de internet (Kollock, 1999).

Dada su importancia, en los entornos virtuales la confianza ha sido ampliamente estudiada (Childers, Carr, Peck y Carson, 2001; Flavián y Guinalíu, 2006; Luna y Velasco, 2005; Sanz, Ruiz y Pérez, 2009; Sundararajan, 2016; Van der Heijden, Verhagen y Creemers, 2003). Al igual que ha ocurrido con la e-reputation (BenítezEyzaguirre, 2016; Márquez-González y Caro, 2017; Medina, 2017; Moya, y Majó, 2017; Pulido y Benítez- Eyzaguirre, 2016), es decir, con ese elemento de la reputación que se deriva específicamente de los contactos electrónicos (Chun y Davies, 2001, p. 316). De estos estudios se deriva que el concepto de reputación suele aplicarse a ámbitos muy diversos, como instituciones universitarias, países, ciudades, empresas... (Ortiz, Villafañe y Caffarel-Serra, 2018, p. 846). Sin embargo, tanto la confianza como la reputación digital resultan esenciales en el ámbito del consumo colaborativo, ya que permiten disminuir el riesgo de los intercambios entre desconocidos (Brändle, 2017, p. 135).

De esta forma, el consumo colaborativo puede definirse como la actividad entre iguales (P2P) basada en obtener, dar o compartir el acceso a bienes y servicios, coordinados a través de comunidades online de colaboración (Hamari, Sjöklint y Ukkonen, 2016, p. 4). El consumo colaborativo es ensalzado por sus partidarios como una herramienta de transformación social que refuerza la cohesión, potencia la capacidad de compra de los consumidores y mejora el medioambiente, mientras que sus detractores lo condenan porque supuestamente introduce competencia desleal, promueve el fraude fiscal y distorsiona el mercado de trabajo (Organización de Consumidores y Usuarios, 2017, p. 1).

Por su parte, la economía colaborativa es definida por la Comisión Europea (2016) como "modelos de negocio en los que plataformas online facilitan la creación de espacios de mercado abiertos para el uso temporal de mercancías o servicios ofrecidos a menudo por particulares". Sin embargo, para Slee $(2016$, p. 32) la economía colaborativa se basa en un pequeño número de empresas tecnológicas que se encuentran respaldadas por grandes cantidades de capital riesgo. En definitiva, la economía colaborativa formaría parte, junto a otras modalidades (gig economy, economía circular, etc.) del consumo colaborativo (Belk, 2014, p. 1598; Frenken, 2017, p. 13; Hamari et al., 2016, p. 1; Möhlmann, 2015, p. 195). 
No se puede olvidar que el auge de las plataformas de consumo colaborativo y de la llamada economía colaborativa es gracias a la generalización del acceso a internet y al desarrollo de las Tecnologías de la Información y de la Comunicación (Patiño, Gómez-Álvarez y Plaza, 2017, p. 53). De esta forma, cabe destacar que la economía colaborativa y el consumo colaborativo se basan en el intercambio de bienes y servicios que utilizan la tecnología como canal "para poner en contacto a las personas y avalar su reputación" (Luis, 2015, p. 67), de ahí el interés por su estudio desde el ámbito de la comunicación.

Ambos fenómenos han experimentado un auge (Noguera et al., 2014) a partir de artículos (Algar, 2007), libros (Botsman y Rogers, 2010) o charlas TED (https://www.ted.com/talks/rachel botsman the case for collaborative consumptio n). En 2012 la economía colaborativa se presentaba como una imparable marea sociocultural (Botsman y Rogers, 2012, p. 224) que transformaba la sociedad provocando una auténtica revolución colaborativa (Cañigueral, 2014, p. 40) y que podría llegar a ser tan importante como la Revolución Industrial (Belk, 2014, p. 1599). Así, aunque la actividad económica colaborativa entre iguales ya está muy consolidada se espera que se afiance aún más con la introducción paulatina del internet de las cosas (Rifkin, 2014, p. 313).

Pese a la importancia de este sector económico y de su vinculación con la confianza y la reputación digital para su supervivencia, se podría afirmar que las contribuciones intelectuales de la academia de comunicación al discurso sobre la economía colaborativa son insuficientes (Gregory y Halff, 2017, p. 4). Ante esta situación parece conveniente aumentar la literatura académica existente relacionada con el tema de estudio, pero para ello primero es necesario identificar y conocer las investigaciones ya realizadas.

\section{OBJETIVOS}

El objetivo principal de este trabajo se basa en recopilar, revisar y sintetizar la producción científica existente sobre la confianza y la reputación digital en el ámbito del consumo colaborativo y de la economía colaborativa. En concreto se pretende:

- Conocer la productividad por años, revistas, autores, organizaciones, países e idiomas;

- Examinar los términos y los conceptos utilizados;

- Analizar la calidad científica de los textos y;

- Averiguar si existen diferencias entre los documentos en función de su calidad.

\section{METODOLOGÍA}

Con la finalidad de alcanzar los objetivos indicados anteriormente se realizó un análisis bibliométrico de la producción científica relacionada con la confianza y la reputación digital en el ámbito del consumo colaborativo y de la economía colaborativa. El universo objeto de estudio estuvo conformado por los artículos académicos publicados entre 2004 y 2017 en revistas científicas indexadas en Web of 
Science (WOS), Scopus y Dialnet. Los artículos científicos fueron escogidos como fuente de información para la realización de esta investigación debido a que históricamente han sido considerados como esenciales para la comunicación de la ciencia (Melero y Hernández-San-Miguel, 2014).

El año 2004 fue elegido como punto de partida para la realización de la búsqueda porque, aunque no existe una definición estándar para el concepto economía colaborativa (sharing economy en inglés), el término sharing aplicado a la disciplina económica se utilizó por primera vez en el ámbito académico en un artículo publicado en 2004 por Yochai Benkler en The Yale Law Journal (Katz, 2015, p. 1068). Cabe destacar que en este artículo ya se establecía el intercambio como fenómeno económico dependiente de la tecnología (Benkler, 2004, p. 358). Así, aunque este tipo de economía, que se basa en que los consumidores garanticen acceso temporal a sus activos físicos infrautilizados posiblemente por dinero (Frenken, 2017), se produce desde que existe la humanidad, el cambio clave se origina a partir de la aparición de plataformas en internet donde las personas comienzan a compartir con extraños, frente a lo habitual hasta el momento que era hacerlo únicamente entre familia, amigos y vecinos (Schor, 2014). Asimismo, se localizaron artículos publicados hasta el año 2017 porque la recogida de datos tuvo lugar el 7 de enero de 2018.

Por su parte, Scopus y WOS fueron escogidas por ser las dos bases de datos multinacionales e internacionales de referencia en el ámbito académico (NavarroBeltrá y Martín-Llaguno, 2013, p. 113). Sin embargo, estas bases poseen ciertos sesgos. Con referencia al idioma, destaca que en Scopus el español es una de las lenguas con menor representación si se considera la cantidad de revistas científicas a nivel mundial que publican en este idioma (Grupo Scimago, 2006, p. 145). A su vez, las revistas científicas indexadas en WOS están escritas principalmente en inglés (Franco-López, Sanz-Valero y Culebras, 2016, p. 65). Además, la representación de las Ciencias Sociales en ambas bases de datos es modesta (Navarro-Beltrá y MartínLlaguno, 2013, p. 113). Así, y con la finalidad de solventar estos sesgos, se incluyó Dialnet en la realización del presente estudio, ya que "es uno de los mayores portales bibliográficos del mundo, cuyo principal cometido es dar mayor visibilidad a la literatura científica hispana" y se centra principalmente en las Ciencias Jurídicas, Humanas y Sociales (https://dialnet.unirioja.es/info/ayuda/qe).

La estrategia de búsqueda principal consistió en utilizar las bases de datos WOS, Scopus y Dialnet para localizar determinadas palabras clave en sus artículos. En concreto, estos documentos debían contener, en el título, en el resumen y/o en las palabras clave, como mínimo un concepto de cada uno de los ámbitos de estudio considerados para la realización del presente estudio, a saber: a) economía colaborativa y consumo colaborativo, b) confianza y reputación y c) internet, comercio electrónico, on-line, digitaly web.

En la tabla 1 se pueden observar las ecuaciones de búsqueda empleadas en cada una de las bases de datos. Estas búsquedas reportaron 47 artículos en WOS, 22 en Scopus y 1 en Dialnet. Tras eliminar las repeticiones se obtuvo un total de 49 textos. 
Con el propósito de seleccionar los documentos vinculados con el tema de estudio se procedió a la lectura del título, del resumen, de las palabras clave y, en ocasiones, del texto completo. De esta forma, se seleccionaron los escritos que cumplieron ciertos criterios de inclusión: artículos académicos publicados en revistas científicas que tuviesen en cuenta la confianza o la reputación en el sector del consumo colaborativo y de la economía colaborativa. Esta selección, que fue efectuada por dos investigadores, identificó un total de 44 textos, todos ellos fueron codificados y analizados para la realización de la presente investigación.

Por tanto, se puede afirmar que el porcentaje de ruido encontrado (artículos no relacionados con la temática del estudio) es adecuado, pues es inferior al $20 \%$ (López-Berna, Papí-Gálvez y Martín-Llaguno, 2011, p. 216). Para conocer los documentos finalmente examinados se puede consultar el anexo 1. Así, cabe destacar que el $59,1 \%$ de estos escritos fue localizado en una única base de datos ( $52,3 \%$ en WOS, $4,5 \%$ en Scopus y $2,3 \%$ en Dialnet), mientras que el $40,9 \%$ fue hallado en dos bases de datos (Scopus y WOS).

Tabla 1. Operaciones de búsqueda en función de la base de datos.

\begin{tabular}{|c|c|}
\hline $\begin{array}{l}\text { Bases de } \\
\text { datos }\end{array}$ & Operaciones de búsqueda \\
\hline Scopus & $\begin{array}{l}\text { Documents search: "sharing economy" OR "collaborative consumption" en el campo } \\
\text { "article title, abstract, keywords" AND trust OR reputation en el campo "article title, } \\
\text { abstract, keywords" AND internet OR e-commerce OR ecommerce OR on-line OR online } \\
\text { OR digital OR web en el campo "article title, abstract, keywords" } \\
\text { Date range (inclusive): Published } 2004 \text { - to - present } \\
\text { Document type: article }\end{array}$ \\
\hline $\begin{array}{l}\text { Web of } \\
\text { Science }\end{array}$ & $\begin{array}{l}\text { Seleccionar una base de datos: todas las bases de datos } \\
\text { Búsqueda básica: "sharing economy" OR "collaborative consumption"en el campo } \\
\text { "tema" AND trust OR reputation en el campos "tema" } A N D \text { internet OR e-commerce OR } \\
\text { ecommerce OR on-line OR online OR digital OR web en el campo "tema" } \\
\text { Periodo de tiempo, desde } 2004 \text { hasta } 2017 \\
\text { Tras la realización de esta búsqueda, los textos mostrados se limitaron por el tipo de } \\
\text { documento y se escogió la opción article }\end{array}$ \\
\hline $\begin{array}{l}\text { Dialnet } \\
\text { plus }\end{array}$ & $\begin{array}{l}\text { Buscar - Buscar documentos: artículos de revista } \\
\text { Contiene las palabras: ("economia colaborativa" OR "consumo colaborativo") AND } \\
\text { (confianza OR reputacion) AND (Internet OR "comercio electronico" OR e-commerce OR } \\
\text { ecommerce OR on-line OR online OR digital OR web) } \\
\text { Publicados entre: } 2004-2017 \\
\text { Tras la realización de esta búsqueda inicial se comprobó si las palabras clave estaban } \\
\text { incluidas en el título, en el resumen o en las palabras clave de manera manual. }\end{array}$ \\
\hline
\end{tabular}

Fuente: elaboración propia.

El protocolo de codificación utilizado para examinar los artículos seleccionados estuvo conformado por tres grandes bloques que abarcaban un total de 35 variables. En concreto, se recogió información sobre: características del artículo (doi, título, palabras clave, idioma, revista de publicación, número y/o volumen de la revista, año de publicación y base de datos), autores y sus organizaciones (nombre y apellido de cada uno de los firmantes, número y sexo de los autores, institución a la que 
pertenecen, número de organizaciones involucradas en cada artículo y país de la organización) y calidad del documento (número de citas e indexación en SJR y JCR).

Con el propósito de analizar la información recolectada se creó una base de datos en el programa informático SPSS versión 24. En concreto, para la descripción de las variables se recurrió a los descriptivos, a las frecuencias, a los gráficos de línea, a las tablas de respuesta múltiple y a las tablas de contingencia. Además, para averiguar la asociación entre variables se utilizó la prueba U de Mann-Whitney cuando la variable dependiente era cuantitativa y el test Chi-cuadrado de Pearson cuando la variable dependiente era cualitativa. En ambos casos el nivel de significación fijado para el análisis estadístico fue $\alpha=0,05$.

\section{RESULTADOS}

A pesar de que la recogida de datos se inicia en el año 2004, el primer documento encontrado data de 2012. A partir de ese momento, y tal y como se puede observar en el gráfico 1, la producción científica relacionada con el tema de estudio se va incrementando con el paso del tiempo. De esta forma, el último año examinado (2017) llama especialmente la atención, ya que cuenta con más de la mitad de los documentos estudiados $(54,5 \%)$. Además, este se sitúa a gran distancia del segundo año más prolífico (2016), pues este únicamente posee el $18,2 \%$ de los artículos. Ante estos datos podría afirmarse que se trata de un tema de reciente interés científico.

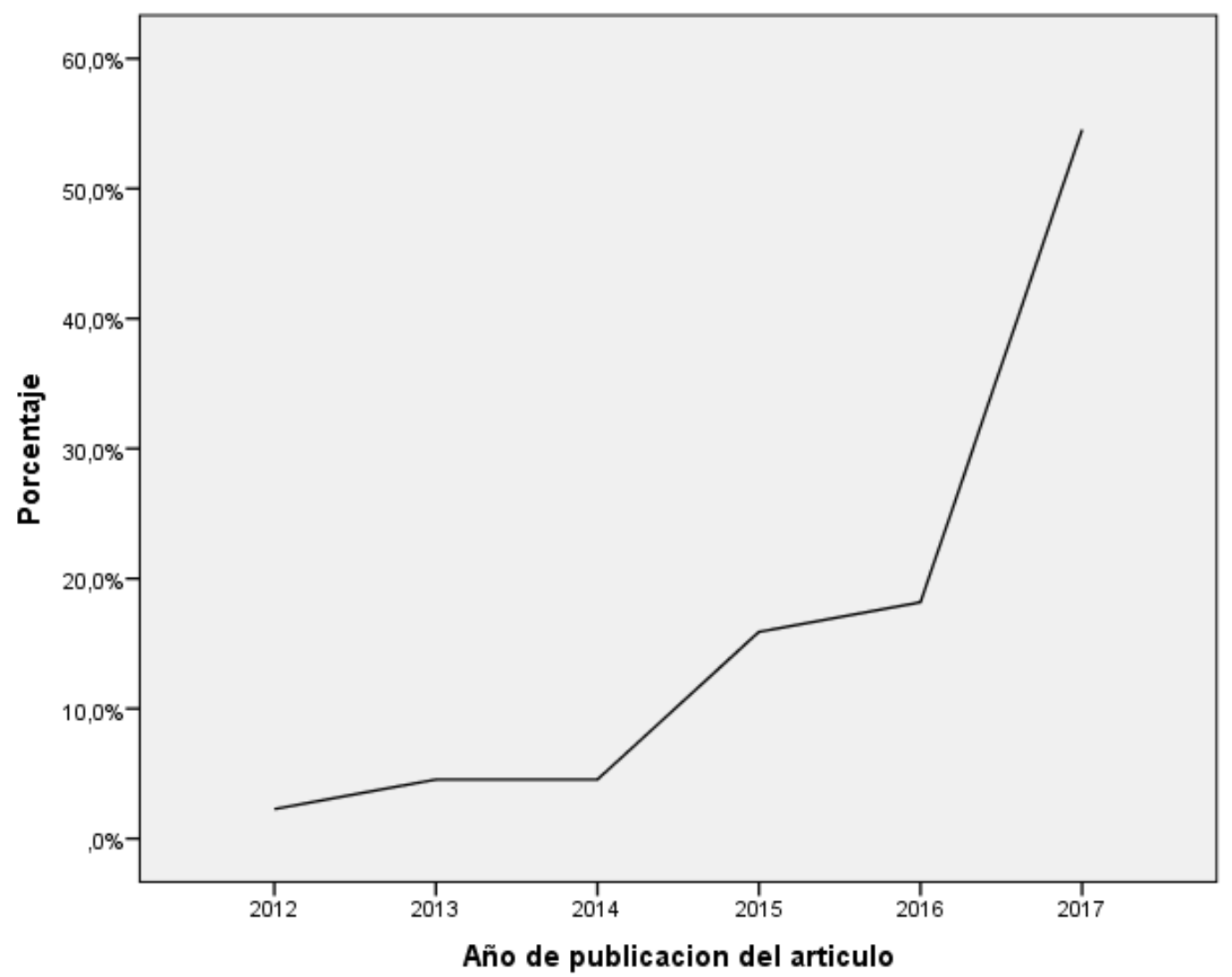

Gráfico 1: año de publicación del artículo.

Fuente: elaboración propia. 
Con referencia a las palabras clave, cabe destacar que en los artículos analizados se han encontrado un total de 429 términos. Como se puede observar en la imagen 1 , que se realizó en el sitio web https://www.nubedepalabras.es/, los más utilizados son: economy $(9,09 \%$ del total), sharing $(8,16 \%)$, consumption $(3,03 \%)$, collaborative $(2,56 \%)$, trust $(2,56 \%)$, digital $(1,86 \%)$ y social $(1,86 \%)$. El uso prioritario de estas palabras se corresponde con los tres ámbitos de estudio considerados para la realización de la presente investigación -a) economía colaborativa y consumo colaborativo, b) confianza y reputación y c) Internet, comercio electrónico, on-line, digital y web-. El resto de términos hallados aparecen con una frecuencia inferior al $1,4 \%$ de las ocasiones.

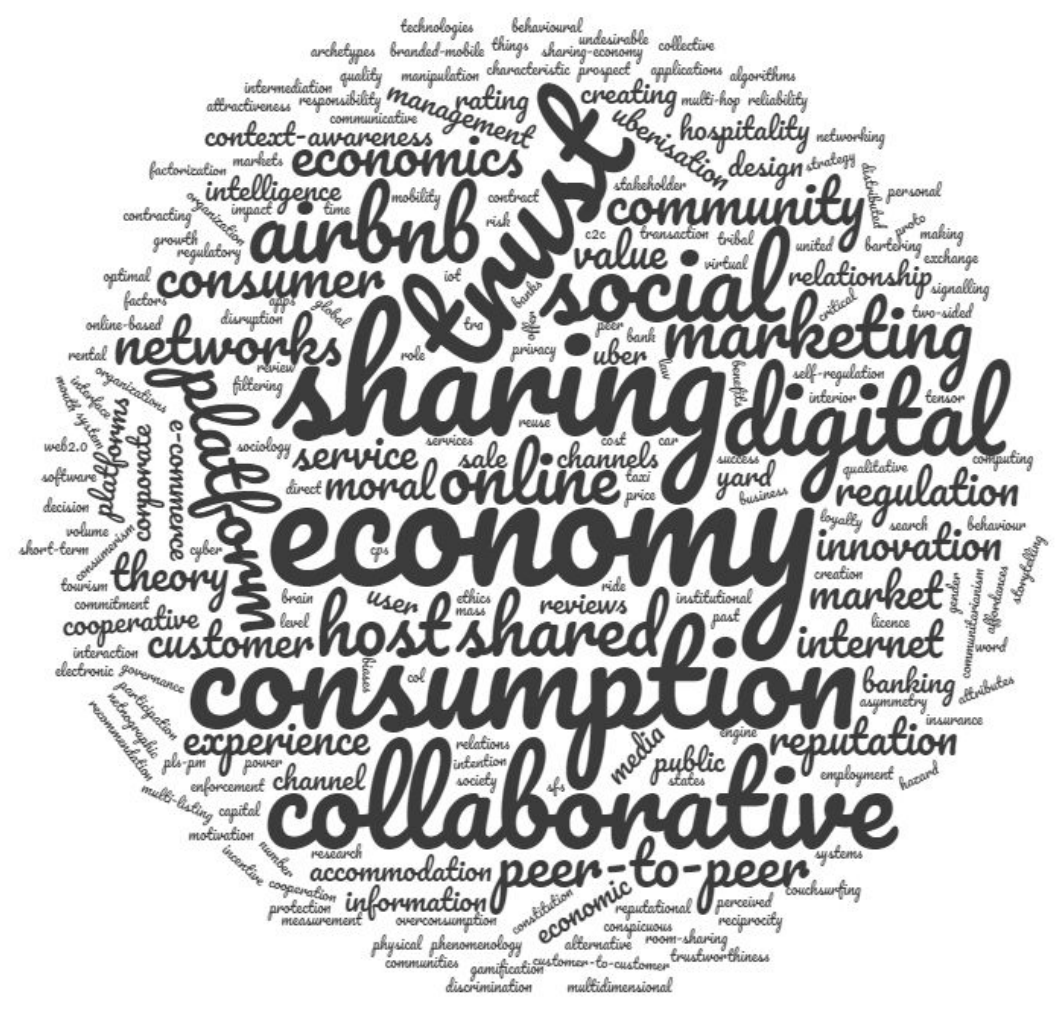

Imagen 1: términos empleados en las palabras clave de los artículos examinados.

Fuente: elaboración propia.

Por otra parte, los 44 escritos analizados han sido hallados en un total de 34 revistas científicas, de manera que la mayoría de ellas $(56,82 \%)$ publica únicamente un artículo. Así, la media de documentos por revista es de 1,3. Esta situación evidencia la variedad de cabeceras que muestran interés en el tema de estudio y la falta de especialización existente. Sin embargo, cabe destacar el caso de la International Journal of Contemporary Hospitality Management, pues publica tres de los textos examinados $(6,8 \%$ del total). Por su parte, las revistas que poseen dos artículos (4,5\%) son: Journal of Consumer Behaviour, Tourism Management, Technological Forecasting and Social Change, Journal of Services Marketing, Journal of Marketing Channels, Annals of Tourism Research, Journal of Law \& Economic Regulation y Journal of Consumers Studies. 
Como era de esperar, predomina la publicación de artículos en lengua inglesa (77,3\% del total). A continuación, aunque a gran distancia, se sitúa el coreano $(15,9 \%)$, mientras que el resto de idiomas encontrados (francés, húngaro y español) únicamente se han podido observar en un escrito $(2,3 \%)$.

El conjunto de los artículos examinados reporta un total de 99 autores y el índice de coautoría, es decir, la media de autores por artículo, es de 2,25. De esta forma, la mayoría de los documentos ( $34,1 \%$ del total) poseen un solo firmante, seguidamente se encuentran los artículos redactados por tres autores (31,8\% del total) y a continuación se pueden observar los que han sido escritos por dos $(20,5 \%)$. Solamente el $13,6 \%$ de los textos cuenta con cuatro signatarios. Por tanto, y debido a que el $65,9 \%$ de los artículos se ha realizado en coautoría, se podría afirmar que es frecuente que los investigadores trabajen este tema de forma conjunta.

En este sentido, cabe señalar que la mayoría de los autores publican únicamente un documento. Sin embargo, destaca la presencia de Karen Xie, ya que firma tres de los artículos examinados (en uno de ellos es la autora principal y en los otros dos ocupan la segunda posición). Por su parte, Stuart J. Barnes, Jiang Wu, Jan Mattsson, Alok Gupta, Paolo Parigi y Karen Cook firman dos artículos, todos ellos en coautoría.

Al considerar el sexo de los autores cabe destacar que en el $20,5 \%$ de los documentos no aparece ninguna mujer frente al $29,5 \%$ en el que no se puede observar ningún hombre. No obstante, al examinar el sexo del firmante principal estos porcentajes se invierten, pues es más habitual encontrar a hombres que ocupan esta posición que a mujeres (52,3\% frente a $45,5 \%$. En el $2,3 \%$ restante se desconoce esta información).

Con referencia a los organismos a los que pertenecen los autores, cabe señalar que en el estudio realizado las relaciones institucionales son frecuentes. De esta forma, al considerar los 28 documentos $^{3}$ en los que podrían existir este tipo de relaciones por haber más de un firmante, estas se producen en el $64,3 \%$ de las ocasiones. Así, la media de entidades implicadas en los artículos con más de un signatario es de 1,82, con un mínimo de 1 y un máximo de 3.

Por su parte, la institución más prolífica es Stanford University, pues se puede observar en 8 ocasiones de 99 (8,08\% del total). A continuación, se sitúan Vienna University of Technology, Sejong University y Wuhan University, ya que cada una de ellas aparece 4 veces $(4,04 \%)$. Además, hay 7 universidades que se muestran en 3 ocasiones $(3,03 \%)^{4}$ y 12 que se pueden ver en $2(2,02 \%)^{5}$. El resto de instituciones

\footnotetext{
${ }^{3}$ Podría haber un documento más en el que se produjesen relaciones institucionales, pero no se ha tenido en cuenta para realizar este cálculo porque se desconoce la vinculación institucional de sus autores.

${ }^{4}$ Sapienza Università di Roma, Dongguk University, Hanyang University, Queensland University of Technology, Universidad Complutense de Madrid, University of Denver y Hebrew University of Jerusalem.
} 
únicamente se observan en una ocasión $(1,01 \%)$. Por tanto, y ante estos datos, se podría afirmar que no hay un centro de referencia especializado en el tema de estudio.

Sin embargo, no se produce esta situación al observar las zonas geográficas a las que pertenecen los autores. Así, el estudio de la confianza y la reputación on-line en el ámbito del consumo colaborativo y de la economía colaborativa parece ser un tema que despierta el interés de los investigadores de Estados Unidos, ya que hasta en 20 ocasiones se han podido observar autores de instituciones radicadas en este país (20,2\%). También podría considerarse como un área de estudio relevante en Corea del Sur y la República de China, pues se han encontrado signatarios de estas zonas en 18 y 11 ocasiones respectivamente $(18,18 \%$ y $11,11 \%)$. Por su parte, Austria, Australia y Reino Unido aparecen 6 veces (6,06\%), España y los Países Bajos se muestran en 5 ocasiones (5,05\%), Italia se puede ver en $4(4,04 \%)$, Israel y Alemania en $3(3,03 \%)$ y Dinamarca y Francia en $2(2,02 \%)$. El resto de países solo aparecen una vez $(1,01 \%)$.

La calidad de los artículos frecuentemente es evaluada en función de si la revista de publicación se encuentra, o no, indexada en determinadas bases de datos. Así, cabe destacar que los documentos analizados podrían considerarse de calidad, ya que el $61,4 \%$ pertenece a revistas que estaban indexadas en Journal Citation Reports (JCR) el año de publicación del artículo, cifra que aumenta hasta el $72,7 \%$ al observar la base de datos Scimago Journal\&Country Rank (SJR).

La calidad de estos artículos se evidencia aún más al considerar el cuartil de estas publicaciones. Así, y debido a que se asigna un cuartil para cada una de las categorías temáticas en las que se inserta la revista, en la base de datos JCR se examinan un total de 47 casos. De esta forma, el $55,3 \%$ forma parte del Q1, el $27,7 \%$ se ubica en el Q2, el $12,8 \%$ en el Q3 y el $4,3 \%$ en el Q4. Por su parte, al estudiar los 65 casos de SJR se puede observar una situación muy similar a la anterior, ya que el cuartil más frecuente vuelve a ser el $1(64,6 \%)$, a continuación se sitúan el Q2 (27,7\%), el Q4 (4,6\%) y el Q3 (3,1\%).

Habitualmente también se suele considerar que el número de citas es un indicador de la calidad de los artículos. Sin embargo, los documentos examinados no se citan con frecuencia, ya que el $56,8 \%$ no posee ninguna cita en WOS, aunque esta cifra se reduce al $27,3 \%$ al observar la base de datos Scopus. Quizás esta situación pueda ser explicada por la reciente publicación de la mayoría de los textos estudiados, de manera que es probable que no hayan tenido tiempo suficiente para obtener un mejor resultado en este indicador de calidad.

En este sentido, cabe destacar que la media de citas por artículo según WOS es de 3,69 (con un mínimo de 0 y un máximo de 45), mientras que en Scopus este

\footnotetext{
${ }^{5}$ University of Applied Sciences Utrecht, Utrecht University, Roskilde University, King's College London, Wuhan University, Korea University, Université Paris Nanterre, Donghua University, The University of Queensland, Hong Kong Polytechnic University, Hongik University y Sookmyung Women's University.
}

Revista de Comunicación de la SEECI. 15 marzo, 2020 / 15 julio, 2020, n 51, 83-107 
valor es de 6,56 (con un mínimo de 0 y un máximo de 56) (ver tabla 2). En ambas bases de datos el documento más citado es el realizado por Möhlmann en 2015.

Tabla 2: citas de los artículos examinados.

\begin{tabular}{|l|l|l|l||l|l|l|l|}
\hline \multicolumn{3}{|l||}{ Citas de los artículo que aparecen en WOS } & \multicolumn{4}{|l|}{ Citas de los artículos que aparecen en Scopus } \\
\hline $\begin{array}{l}\text { Número } \\
\text { de citas }\end{array}$ & Frecuencia & Porcentaje & $\begin{array}{l}\text { Porcentaje } \\
\text { acumulado }\end{array}$ & $\begin{array}{l}\text { Número } \\
\text { de citas }\end{array}$ & Frecuencia & Porcentaje & $\begin{array}{l}\text { Porcentaje } \\
\text { acumulado }\end{array}$ \\
\hline 0 & 25 & 59,5 & 59,5 & 0 & 12 & 37,5 & 37,5 \\
\hline 1 & 4 & 9,5 & 69 & 1 & 5 & 15,6 & 53,1 \\
\hline 2 & 5 & 11,9 & 81 & 2 & 5 & 15,6 & 68,8 \\
\hline 3 & 2 & 4,8 & 85,7 & 3 & 2 & 6,3 & 75 \\
\hline 6 & 1 & 2,4 & 88,1 & 4 & 2 & 6,3 & 81,3 \\
\hline 7 & 1 & 2,4 & 90,5 & 14 & 2 & 6,3 & 87,5 \\
\hline 13 & 1 & 2,4 & 92,9 & 19 & 1 & 3,1 & 90,6 \\
\hline 27 & 1 & 2,4 & 95,2 & 30 & 1 & 3,1 & 93,8 \\
\hline 37 & 1 & 2,4 & 97,6 & 48 & 1 & 3,1 & 96,9 \\
\hline 45 & 1 & 2,4 & 100 & 56 & 1 & 3,1 & 100 \\
\hline TOTAL & 42 & 100 & & TOTAL & 32 & 100 & \\
\hline
\end{tabular}

Fuente: elaboración propia.

A pesar de todos estos datos, no se puede olvidar que la academia suele considerar a los documentos indexados en JCR como los de mayor impacto y calidad. Ante esta situación parece relevante conocer si existen diferencias entre los artículos indexados en esta base de datos y los que no están.

En este sentido, cabe señalar que no suelen existir diferencias estadísticamente significativas en los artículos en función de si estos están, o no, indexados en la base de datos JCR. De hecho, no se observan desemejanzas en los años, en los autores, en el número de signatarias, en el sexo del primer firmante, en las instituciones a las que estos pertenecen, en los países ni en las citas recibidas en Scopus.

Sin embargo, sí existen diferencias en el idioma, de forma que todos los documentos recopilados en la base de datos JCR están redactados en lengua inglesa $\left(x^{2}=20,554 ; p=0,000\right)$. Además, el rango promedio del total de autores por artículo es mayor en los documentos indexados en JCR que en los no indexados $(25,57$ frente a 17,62) $(U=146,500 ;$ sig. $=0,037)$, situación que también se puede observar en los signatarios masculinos $(25,94$ vs. 15,34$)(U=109,500 ;$ sig. $=0,005)$, en el número de instituciones $(25,59$ frente a 15,94$)(U=119,000$; sig.= 0,006) y en la cantidad de citas que reciben los textos en la base de datos Web of Science $(26,00 \mathrm{y}$ $14,19)(U=91,000$; sig. $=0,001)$. Por tanto, se podría afirmar que los artículos publicados en revistas indexadas en JCR se caracterizan por estar redactados en inglés, por tener una mayor colaboración, tanto a nivel de autores como de instituciones, y por contar con más citas en WOS. 
Navarro-Beltrá, M. y Martínez-Polo, J.

Estudio bibliométrico sobre reputación digital y economía colaborativa (2004-2017)

\section{DISCUSIÓN/CONCLUSIONES}

Los datos obtenidos en este trabajo ponen de manifiesto que el interés académico por la confianza y la reputación digital en el sector del consumo colaborativo y de la economía colaborativa es reciente. Esta situación era de esperar, ya que no se puede olvidar que tanto la e-reputación (Mababu Mukiur, 2016, p. 148) como la economía colaborativa (Pimentel, 2017, pp. 108-109) son fenómenos nuevos. A pesar de su reciente aparición, la reputación digital es un concepto que ha adquirido gran relevancia, especialmente desde que internet y los social media son importantes fuentes de información para la sociedad (MadrigalMoreno, Arroyo-Cañada y Gil-Lafuente, 2017, p. 47). Por tanto, y ante esta situación, se espera que la producción científica relacionada con el tema de estudio siga aumentando en los próximos años.

Dada la importancia de la materia, parece relevante destacar que además la literatura académica relacionada con el tema de estudio es de calidad, ya que gran parte de los artículos examinados se publican en revistas indexadas en cuartiles (Q) preferentes de JCR y SJR. A pesar de que el cuartil al que pertenece la revista es un elemento importante que contribuye a determinar su calidad (Ordóñez y Sierra, 2018 , p. 49), no se puede olvidar que esta asignación depende del factor de impacto, que se calcula a partir del total de citas que obtiene una publicación y no todos los artículos reciben la misma cantidad (Sobrido y Sobrido, 2013, p. 266). Por tanto, hay autores que afirman que el factor de impacto solo se debería de utilizar para valorar la calidad de revistas, no de artículos concretos (Aleixandre-Benavent, Valderrama-Zurián, González de Dios y de Granda-Orive, 2004), para ello se debería de hacer alusión al índice de citas (Sobrido y Sobrido, 2013, p. 266). A pesar de que el número de citas de los artículos examinados es reducido, esta situación podría explicarse fácilmente por su reciente publicación.

Por otra parte, cabe destacar que no se ha podido encontrar una revista, un autor, ni un centro de investigación de referencia. No obstante, sí existe una zona geográfica que presta especial atención al tema de estudio, pues los firmantes de los documentos estudiados suelen pertenecer a instituciones radicadas en Estados Unidos. Esta situación también era de esperar, ya que se trata de "la principal potencia científica" (Castañeda y Jiménez, 2017, p. 29). Además, la mayoría de los artículos son redactados en inglés, que es considerado el idioma científico por excelencia (Téllez-Zenteno, Morales-Buenrostro y Estañol, 2007, pp. 485, 487).

Los datos recabados en el presente estudio coinciden con los resultados obtenidos en anteriores investigaciones que analizan el ámbito de la economía colaborativa en general, de manera que se podría concluir afirmando que se trata de un tema que recientemente ha captado el interés de los investigadores (Chen, 2016, p. 62; Durán-Sánchez, Álvarez-García, del Río-Rama y Malonado-Erazo, 2016, pp. 11-13), que no existen revistas especializadas en el tema de estudio, que Estados Unidos es el país más prolífico y que lo más habitual es encontrar un autor por publicación (Durán-Sánchez, Álvarez-García, del Río-Rama y Malonado-Erazo, 2016, pp. 11-13). 
Finalmente, cabe indicar que la principal limitación de la presente investigación se basa en haber examinado únicamente artículos académicos relacionados con el tema de estudio indexados en Scopus, WOS y Dialnet. A pesar de haber analizado solamente una muestra de la literatura académica existente, los datos obtenidos contribuyen a ampliar el conocimiento existente sobre la producción científica relacionada con la confianza y la reputación digital en el ámbito de la economía y del consumo colaborativo. De esta forma, se abre una posible línea de investigación futura centrada en examinar revistas incluidas en otras bases de datos. También sería interesante comprobar el interés académico en la confianza y la reputación online en otras disciplinas.

\section{REFERENCIAS}

Aced, C. (2010). Perfiles profesionales 2.0 [Professional profiles 2.0]. Barcelona, Spain: Editorial UOC.

Aced, C. (2018). Relaciones públicas 2.0. Cómo gestionar la comunicación corporativa en el entorno digital [Public relations 2.0. How to manage corporate communication in the digital environment]. Barcelona, Spain: Editorial UOC.

Aleixandre-Benavent, R., Valderrama-Zurián, J. C., González de Dios, J. y de GrandaOrive, J. I. (2004). El factor de impacto. Un polémico indicador de calidad científica [The impact factor. A controversial indicator of scientific quality]. Revista Española de Economía de la Salud, 3(5), 242-249.

Algar, R. (2007). Collaborative consumption. Leisure Report, 4, 72-83. Retrieved from https://www.oxygen-consulting.co.uk/insights/collaborative-consumption/

Belk, R. (2014). You are what you can access: Sharing and collaborative consumption online. Journal of Business Research, 678), 1595-1600. doi: https://doi.org/10.1016/j.jbusres.2013.10.001

Benítez-Eyzaguirre, L. (2016). Análisis de la recomendación entre iguales en la reputación online de las organizaciones [Analysis of peer recommendations in the online reputation of organizations]. El Profesional de la Información, 25(4), 652660. doi: http://dx.doi.org/10.3145/epi.2016.jul.15

Benkler, Y. (2004). Sharing nicely: on shareable goods and the emergence of sharing as a modality of economic production. The Yale Law Journal, 114(2), 273-358. doi: http://dx.doi.org/10.2307/4135731

Botsman, R., \& Rogers, R. (2011). What's mine is yours: how collaborative consumption is changing the way we live. New York, United States: HarperCollins Business.

Brändle, G. (2017). Controversias en torno al impacto social del consumo colaborativo: algunos elementos para la discusión [Controversies around the social 
impact of collaborative consumption: some elements for discussion]. Redes.com: Revista de Estudios para el Desarrollo Social de la Comunicación, 15, 122-143. doi: http://dx.doi.org/10.15213/redes.n15.p123

Cañigueral, A. (2014). Vivir mejor con menos. Descubre las ventajas de la nueva economía colaborativa [Live better with less. Discover the advantages of the new sharing economy]. Barcelona, Spain: Penguin Random House.

Castañeda, L. A. y Jiménez, D. (2017). Impacto de la migración de recursos humanos en el desarrollo de la ciencia y tecnología de México [Impact of the migration of human resources in the development of science and technology in Mexico]. Revista Ciencia Administrativa, 2, 25-32. Retrieved from https://www.uv.mx/iiesca/files/2018/07/VOLUMEN2.pdf\#page=27

Castells, M. (2005). La era de la información: economía, sociedad y cultura. La sociedad red. [The information age: economy, society and culture Volume I: the rise of the network society] (Vol. 1). Madrid, Spain: Alianza.

Castillo, A. (2009). Relaciones públicas. Teoría e historia [Public relations. Theory and history]. Barcelona, Spain: Editorial UOC.

Cheng, M. (2016). Sharing economy: A review and agenda for future research. International Journal of Hospitality Management, 57, 60-70. doi: https://doi.org/10.1016/j.ijhm.2016.06.003

Childers, T. L., Carr, C. L., Peck, J., \& Carson, S. (2001). Hedonic and utilitarian motivations for online retail shopping behavior. Journal of Retailing, $7 \pi(4), 511-$ 535. doi: https://doi.org/10.1016/S0022-4359(01)00056-2

Chun, R., \& Davies, G. (2001). E-reputation: the role of mission and vision statements in positioning strategy. Journal of Brand Management, 8(Spring), 315333. doi: https://doi.org/10.1057/palgrave.bm.2540031

Comisión Europea (2016). Una Agenda Europea para la economía colaborativa [A European Agenda for the collaborative economy]. Retrieved from https://tinyurl.com/y3y8xugv

Durán-Sánchez, A., Álvarez-García, J., del Río-Rama, M. C., y Malonado-Erazo, C. P. (2016). Economía colaborativa: análisis de la producción científica en revistas académicas [Collaborative economy: Analysis of scientific production in academic magazines]. Revista de Gestão e Secretariado - GeSec, 7(3), 1-20. https://doi.org/10.7769/gesec.v7i3.617

Flavián, C. y Guinalíu, M. (2006). La confianza y el compromiso en las relaciones a través de Internet. Dos pilares básicos del marketing estratégico en la red [Trust and commitment in relationships through the Internet. Two basic pillars of 
strategic marketing in the net]. Cuadernos de Economía y Dirección de la Empresa, (29), 133-160. Retrieved from https://tinyurl.com/yy2okjh6

Franco-López, Á., Sanz-Valero, J. y Culebras, J. M. (2016). Publica en castellano, o en cualquier otro idioma que no sea inglés, negativo para el factor de impacto y citaciones [To publish in Spanish or in any non English language, negative for impact factor and citations]. Journal of Negative \& No Positive Results, 1(2), 6570. doi: https://doi.org/10.19230/jonnpr.2016.1.2.1005

Frenken, K. (2017). Political economies and environmental futures for the sharing economy en Philosophical Transactions of the Royal Societ. Philosophical Transactions of the Royal Society A, 375(2095).

doi: http://dx.doi.org/10.1098/rsta.2016.0367

Fuentes, R. (2018). Filantropía y empresas vitivinícolas. Gestión de la información desde los medios sociales 2.0 [Philanthropy and the wine sector. Information management through social media 2.0]. Obra Digital, 15, 13-29. Retrieved from https://www.raco.cat/index.php/ObraDigital/article/view/340680/431751

Gregory, A., \& Halff, G. (2017). Understanding public relations in the 'sharing economy'. Public Relations Review, 43(1), 4-13.

doi: https://doi.org/10.1016/j.pubrev.2016.10.008

Grunig, J. E., \& Hunt, T. (2000). Dirección de Relaciones Públicas [Public Relations Management]. Barcelona, Spain: Ediciones Gestión 2000.

Grupo Scimago (2006). Análisis de la cobertura de la base de datos Scopus [Analysis of Scopus database coverage]. El Profesional de la Información, 15(2), 144-145. Retrieved from https://tinyurl.com/yy55osga

Hamari, J., Sjöklint, M., \& Ukkonen, A. (2016). The sharing economy: Why people participate in collaborative consumption. Journal of the Association for Information Science and Technology, 67(9), 2047-2059. doi: https://doi.org/10.1002/asi.23552

Katz, V. (2015). Regulating the sharing economy. Berkeley Technology Law Journal, 30(4), 1067-1126. doi: http://dx.doi.org/10.15779/Z38HG45

Kollock, P. (1999). The production of trust in online markets. Advances in Group Processes, 16(1), 99-123.

López-Berna, S., Papí-Gálvez, N., y Martín-Llaguno, M. (2011). Productividad científica en España sobre las profesiones de comunicación entre 1971 y 2009 [Scientific productivity in Spain concerning communications-related professions between 1971 and 2009]. Revista Española de Documentación Científica, 34(2), 212-231. doi: http://dx.doi.org/10.3989/redc.2011.2.801 
Luis, M. (2015). Innovación social: el porqué de un fenómeno emergente [Social Innovation and how it is arriving into society]. Oikonomics: Revista de Economía, empresa y Sociedad, 3, 66-72. Retrieved from https://tinyurl.com/y5z2lnlv

Luna, M. y Velasco, J. L. (2005). Confianza y desempeño en las redes sociales [Trust and performance in social networks]. Revista Mexicana de Sociología, 67(1), 127162. doi: http://dx.doi.org/10.22201/iis.01882503p.2005.001.6014

Mababu Mukiur, R. (2016). Reclutamiento a través de las redes sociales: Reclutamiento 3.0 [Recruitment through social media: Recruitment 3.0]. Opción, 32(10), 135-151. Retrieved from https://tinyurl.com/yxkfa3x9

Madrigal-Moreno, S., Arroyo-Cañada, F. J. y Gil-Lafuente, J. (2017). Análisis de los retos del desarrollo sostenible de Barcelona como Smart City mediante el estudio de su reputación online [Analysis of the challenges of the sustainable development of Barcelona as Smart City through the study of its online reputation]. Cuadernos del CIMBAGE, 2(19), 29-50. Retrieved from https://tinyurl.com/y33g4dsm

Maestro, L., Cordón, D. y Abuín, N. (2018). La comunicación publicitaria en entornos digitales: herramientas para garantizar la reputación corporativa [Corporate communication in digital environments: tools to guarantee coporate reputation]. Prisma Social, 22, 209-228. Retrieved from http://revistaprismasocial.es/article/view/2582/2759

Márquez-González, C. y Caro, J. L. (2017). Ciudades Patrimonio de la Humanidad de España: la reputación online como elemento de desarrollo turístico [World Heritage Cities of Spain: eWOM as an element of tourism development]. Revista de Turismo y Patrimonio Cultura, 15(2), 437-457. Retrieved from http://www.pasosonline.org/es/lectores/ultimo-numero/1062-1

Medina, P. (2017). La gestión de la reputación online de las marcas hospitalarias: una propuesta de modelo [The online reputation management of hospital brands: a model proposal]. Zer, 22(43), 53-68. doi: http://dx.doi.org/10.1387/zer.17908

Melero, R. y Hernández-San-Miguel, J. (2014). Acceso abierto a los datos de investigación, una vía hacia la colaboración científica [Open access to research data: a track towards scientific collaboration]. Revista Española de Documentación Científica, 374). doi: http://dx.doi.org/10.3989/redc.2014.4.1154

Miyashiro, H. (2017). Planificación estratégica de relaciones públicas y la reputación corporativa en el corporate empresarial peruano [Strategic planning of public relations and corporate reputation in the Peruvian corporate business]. Cultura, 31, 87-113. doi: https://doi.org/10.24265/cultura.2017.v31.05

Möhlmann, M. (2015). Collaborative consumption: determinants of satisfaction and the likelihood of using a sharing economy option again. Journal of Consumer Behaviour, 14(3), 193-207. doi: https://doi.org/10.1002/cb.1512 
Moya, D. H. y Majó, J. (2017). Análisis de comentarios en redes sociales para mejorar la reputación online hotelera [Analysis from reviews in social media to improve hotel's online reputation]. Turismo y Sociedad, 20, 169-190. doi: https://doi.org/10.18601/01207555.n20.09

Mut M. (2012). La desvirtualización del concepto reputación en el entorno virtual [The devirtualization of the reputation concept in the virtual environment]. adComunica. Revista Científica de Estrategias, Tendencias e Innovación en Comunicación, 3, 227-229. doi: http://dx.doi.org/10.6035/2174-0992.2012.3.18

Navarro-Beltrá, M. y Martín-Llaguno, M. (2013). Análisis bibliométrico de la investigación sobre mujer y publicidad: diferencias en medios impresos y audiovisuales [Bibliometric Analysis of Research on Women and Advertising: Differences in Print and Audiovisual Media]. Comunicar, 21(41), 105-114. doi: http://dx.doi.org/10.3916/C41-2013-10

Noguera, J. M., Martínez, J., Nicolás, M. A., Pérez, M., Gómez, A., Grandío, M. M., Hernández, F. y Del Henar, P. (2014). Economía de la participación [Participation Economy]. Madrid, Spain: Fundación EOI.

Organización de Consumidores y Usuarios (Coord.) (2016). Collaboration or business? Collaborative consumption: from value for users to a society with values. España: OCU Ediciones, S. A.

Ordóñez, J. B. y Sierra, M. M. (2018). El núcleo básico de las publicaciones periódicas: una metodología para su identificación. El caso del Instituto de Ciencias Nucleares, UNAM [The periodicals core collection: a methodology for their identification. The case of the Institute of Nuclear Sciences, National Autonomous University of Mexico]. Biblioteca Universitaria, 21(1), 41-54. doi: http://dx.doi.org/10.22201/dgb.0187750xp.2018.1.199

Ortiz, Y., Villafañe, J. y Caffarel-Serra, C. (2018). Investigación para la evaluación de la reputación de los medios de comunicación [Research for the assessment of media reputation]. Revista Latina de Comunicación Social, 73, 845-869. doi: http://dx.doi.org/10.4185/RLCS-2018-1285

Patiño, D., Gómez-Álvarez, R. y Plaza-Angulo, J. (2017). Caracterización económica y cuantificación de la economía colaborativa [Economic characterization and quantification of the sharing economy]. In M. Rodríguez-Piñero \& M. HerándezBejarano (Dir.), Economía colaborativa y trabajo en plataforma: realidades y desafíos [Sharing economy and platform work: realities and challenges] (pp. 51101). Albacete, Spain: Bomarzo.

Pimentel, G. L. (2017). La personalización de las relaciones económicas a través de la economía colaborativa: los límites al supuesto de no-tuismo [The personalization of economic relations through the sharing economy: the limits to the assumption of non-tuism]. Revista Académica ECO, 17, 107-115. Retrieved from https://tinyurl.com/y4hqvxgr 
Piñeiro-Otero, T. y Martínez-Rolán, X. (2016). Los memes en el activismo feminista en la Red. \#ViajoSola como ejemplo de movilización transnacional [Memes in the Internet feminist activism. \#ViajoSola as an example of transnational mobilization]. Cuadernos.info, 39, 17-37.

doi: http://dx.doi.org/10.7764/cdi.39.1040

Pulido, M. y Benítez-Eyzaguirre, L. (2016). "Recomendación entre iguales": el papel de los prosumidores en la reputación online de las organizaciones ['Peer recommendation': the role of prosumers in the online corporate reputation]. Pensar la Publicidad, 10, 49-62. doi: http://dx.doi.org/10.5209/PEPU.53773

Rifkin, J. (2014). La sociedad de coste marginal cero. El Internet de las cosas, el procomún colaborativo y el eclipse del capitalismo [The zero marginal cost society. The Internet of things, the collaborative commons and the eclipse of capitalism]. Barcelona, Spain: Espasa.

Rivero, M. (2017). Redimensionamiento del área de Comunicación de la Universidad La Salle Cancún [Proposal for a Department of Public Relations for the University La Salle Cancun]. Revista Internacional de Relaciones Públicas, 713), 161-180. doi: http://dx.doi.org/10.5783/RIRP-13-2017-10-161-180

Sánchez-Alzate, J. A. y Montoya, L. A. (2016). Factores que afectan la confianza de los consumidores por las compras a través de medios electrónicos [Factors affecting the consumer trust for shopping through electronic media]. Pensamiento y Gestión, 40, 159-183. doi: http://dx.doi.org/10.14482/pege.40.8809

Sánchez-Alzate, J. A. y Montoya, L. A. (2017). La confianza como elemento fundamental en las compras a través de canales de comercio electrónico. Caso de los consumidores en Antioquia, Colombia. Innovar, 27(64), 11-22. doi: https://doi.org/10.15446/innovar.v27n64.62365

Sanz, S., Ruiz, C. y Pérez, I. (2009). Conceptos, dimensiones y antecedentes de la confianza en entornos virtuales [Concepts, dimensions and background of trust in virtual environments]. Teoría y praxis, 6, 31-56. Retrieved from https://www.redalyc.org/articulo.oa?id $=456145109003$

Schor, J. (2014). Debating the sharing economy. Great transition initiative: Toward a transformative vision and praxis. Retrieved from https://tinyurl.com/mqnv349

Sellas, T. (2014). El podcasting en las relaciones públicas: la voz de las organizaciones en la web 2.0 [Podcasting in public relations: the voice of organizations in the web 2.0]. In D. Fernández-Quijada y M. Ramos-Serrano (Eds.), Tecnologías de la persuasión: uso de las TIC en publicidad y relaciones públicas [Persuasion technologies: use of ICT in advertising and public relations] (pp. 197-214). Barcelona, Spain: Editorial UOC.

Slee, T. (2016). Lo tuyo es mío [What's yours is mine]. Barcelona, Spain: Taurus. 
Sobrido, N. y Sobrido M. (2013). ¿Se puede evaluar la calidad de las revistas científicas? Principales limitaciones [It is possible to assess the quality of scientific journals? Major constraints]. Enfermería Global, 12(3), 265-272. Retrieved from https://revistas.um.es/eglobal/article/view/eglobal.12.3.165471/150011

Sundararajan, A. (2016). The sharing economy: The end of employment and the rise of crowd-based capitalism. Cambridge, United States: Mit Press.

Téllez-Zenteno, J. F., Morales-Buenrostro, L. E. y Estañol, B. (2007). Análisis del factor de impacto de las revistas científicas latinoamericanas [Impact factor of Latin American medical journals]. Revista Médica de Chile, 135(4), 480-487. doi: doi: http://dx.doi.org/10.4067/S0034-98872007000400010

Valdez, A., Borrayo, C. y Muñoz, M. (2018). Las relaciones públicas y la comunicación en los desastres naturales. El caso del sismo del 19 de septiembre de 2017 en México [Public relations and communication in natural disasters: The case of the earthquake of September 19, 2017 in Mexico]. Revista Latina de Comunicación Social, 73, 447-461. doi: http://dx.doi.org/10.4185/RLCS-2018-1264

Van der Heijden, H., Verhagen, T., \& Creemers, M. (2003). Understanding online purchase intentions: contributions from technology and trust perspectives. European Journal of Information Systems, 12(1), 41-48. doi: http://dx.doi.org/10.1057/palgrave.ejis.3000445

Zapata-Palacios, L. (2016). Industria de la comunicación y economía digital: Guía básica del Dircom [Communication industry and digital economy: Dircom's basic guide]. Barcelona, Spain: Editorial UOC.

\section{AUTORES:}

\section{Marián Navarro-Beltrá}

Licenciada en Publicidad y RRPP (2008) por la Universidad de Alicante y Doctora Cum Laude (2013) por la misma universidad. Actualmente es profesora e investigadora en la Universidad Católica de Murcia (UCAM) en el área de Publicidad y RRPP. mnavarro2@ucam.edu

Orcid ID: https://orcid.org/0000-0002-6898-569X

Google Scholar: https://scholar.google.es/citations?hl=es\&user= UxZOhAAAAA]

\section{Josep Martínez-Polo}

Profesor e investigador en la Universidad Católica de Murcia (UCAM) en el área de Publicidad y RRPP.

jmmartinez@ucam.edu

Orcid ID: http://orcid.org/0000-0003-0262-0257

Google Scholar: https://scholar.google.es/citations?user=nxaiYpMAAAAJ\&hl=es 


\section{ANEXO 1}

Abrahao, B., Parigi, P., Gupta, A., \& Cook, K. S. (2017). Reputation offsets trust judgments based on social biases among Airbnb users. Proceedings of the National Academy of Sciences, 114(37), 9848-9853.

https://doi.org/10.1073/pnas.1604234114

Balnaves, M. (2012). The Australian finance sector and social media: Towards a history of the new banking. Media International Australia, 143(1), 132-145. https://doi.org/10.1177/1329878X1214300115

Barnes, S. J., \& Mattsson, J. (2016). Building tribal communities in the collaborative economy: an innovation framework. Prometheus, 34(2), 95-113. https://doi.org/10.1080/08109028.2017.1279875

Barnes, S. J., \& Mattsson, J. (2017). Understanding collaborative consumption: Test of a theoretical model. Technological Forecasting and Social Change, 118, 281292. https://doi.org/10.1016/j.techfore.2017.02.029

Calo, R., \& Rosenblat, A. (2017). The taking economy: Uber, information, and power. Columbia Law Review, 11入6), 1623-1690. Retrieved from https://digitalcommons.law.uw.edu/faculty-articles/47

Celata, F., Hendrickson, C. Y., \& Sanna, V. S. (2017). The sharing economy as community marketplace? Trust, reciprocity and belonging in peer-to-peer accommodation platforms. Cambridge Journal of Regions, Economy and Society, 10(2), 349-363. https://doi.org/10.1093/cjres/rsw044

CheonHyejung, K., Siwuel, L., \& Dong, I. (2014). Creating shared value: Redefining the role of the corporation and consumer. Journal of Consumer Studies, 25(3), 120. Retrieved from https://tinyurl.com/y6pb28w5

de Rivera, J., Gordo, Á., Cassidy, P., \& Apesteguía, A. (2017). A netnographic study of P2P collaborative consumption platforms' user interface and design. Environmental Innovation and Societal Transitions, 23, 11-27. http://dx.doi.org/10.1016/j.eist.2016.09.003

Einav, L., Farronato, C., \& Levin, J. (2016). Peer-to-peer markets. Annual review of economics, $8, \quad 615-635$. https://doi.org/10.1146/annurev-economics-080315$\underline{015334}$

Ert, E., Fleischer, A., \& Magen, N. (2016). Trust and reputation in the sharing economy: The role of personal photos in Airbnb. Tourism Management, 55, 62-73. https://doi.org/10.1016/j.tourman.2016.01.013 
Hofmann, E., Hartl, B., \& Penz, E. (2017). Power versus trust-what matters more in collaborative consumption?. Journal of Services Marketing, 31(6), 589-603. https://doi.org/10.1108/JSM-09-2015-0279

Gamito, M. C. (2016). Regulation. com. self-regulation and contract governance in the platform economy: A research agenda. European Journal of Legal Studies, 9(2), 53-67. Retrieved from http://hdl.handle.net/1814/46068

Garrett, A., Straker, K., \& Wrigley, C. (2017). Digital channels for building collaborative consumption communities. Journal of Research in Interactive Marketing, 11(2), 160-184. https://doi.org/10.1108/JRIM-08-2016-0086

Gonzalez-Padron, T. L. (2017). Ethics in the sharing economy: Creating a legitimate marketing channel. Journal of Marketing Channels, 24(1-2), 84-96. http://dx.doi.org/10.1080/1046669X.2017.1347005

Gregory, A., \& Halff, G. (2017). Understanding public relations in the 'sharing economy'. Public Relations Review, 43(1), 4-13. https://doi.org/10.1016/j.pubrev.2016.10.008

Heylighen, F. (2017). Towards an Intelligent Network for Matching Offer and Demand: from the sharing economy to the Global Brain. Technological Forecasting and Social Change, 114, 74-85. https://doi.org/10.1016/j.techfore.2016.02.004

Kaloz, E. (2015). A közösségi gazdaság: elméleti megfontolások és a gyakorlat jellemzői [The community economy - Theoretical considerations and practice characteristics]. Információs Társadalom, 15(1), 44-67. Retrieved from https://matarka.hu/klikk.php?cikkmutat=2374793

Kang, B. J., \& Choi, M. H. (2013). An exploratory study on the prospect and challenges of the sharing economy. Journal of Policy Development, 13(1), 143170. Retrieved from https://tinyurl.com/yzalaloh

Karlsson, L., Kemperman, A., \& Dolnicar, S. (2017). May I sleep in your bed? Getting permission to book. Annals of Tourism Research, 62, 1-12. https://doi.org/10.1016/j.annals.2016.10.002

Key, T. M. (2017). Domains of digital marketing channels in the sharing economy. Journal of Marketing Channels, 24(1-2), 27-38. https://doi.org/10.1080/1046669X.2017.1346977

Kim, J. H., (2017). The effect of AirBnB host characteristics on host trust and reuse intention. Korean Journal of Hospitality and Tourism, 26(7), 55-70. Retrieved from https://tinyurl.com/y64xy5x9 
Kim, S., Tsolmon, B., \& Kim, N. (2017). Users' selection attributes of accommodation sharing platform: Focusing on users of airbnb. Journal of Tourism Studies, 29(2), 51-69. Retrieved from https://tinyurl.com/y426jpu3

Lampinen, A., Huotari, K. J. E., \& Cheshire, C. (2015). Challenges to participation in the sharing economy: the case of local online peer-to-peer exchange in a single parents' network. Interaction Design and Architecture(s) Journal, (24), 16-32. Retrieved from http://mobilelifecentre.org/sites/default/files/24 1.pdf

Lee, H. J. (2017). The nature of innovation in digital platform industry and the proper regulatory response. Administrative Law Journal, 49, 53-74. Retrieved from https://tinyurl.com/yxnx7c4b

Lee, J. Y. (2015). Trust and social commerce. University of Pittsburgh Law Review, 77(2), 137-181. https://doi.org/10.5195/lawreview.2015.395

Liang, S., Schuckert, M., Law, R., \& Chen, C. C. (2017). Be a "Superhost": The importance of badge systems for peer-to-peer rental accommodations. Tourism management, 60, 454-465. https://doi.org/10.1016/j.tourman.2017.01.007

Luckner, N., Fitzpatrick, G., Werner, K., \& Subasi, Ö. (2015). Setting up and running a sharing service: an organisational perspective. Interaction Design and Architecture(s) Journal, (24), 63-80. Retrieved from http://www.mifav.uniroma2.it/inevent/events/idea2010/doc/24 4.pdf

Luis, M. (2015). Innovación social: el porqué de un fenómeno emergente [Social Innovation and how it is arriving into society]. Oikonomics: Revista de Economía, empresa y Sociedad, (3), 66-72. Retrieved from https://tinyurl.com/y5z2Inlv

Marais, B. (2016). Uberizing Regulators?. Journal of Law \& Economic Ragulation, 9(2), 110-127. Retrieved from https://tinyurl.com/y65od2rc

Marais, B., Yoon, S. P., Lim, J. H., \& Kwon, H. Y. (2016). Uberizing Regulators?. Journal of Law \& Economic Regulation, 9(2), 128-142. Retrieved from http://www.dbpia.co.kr/Journal/ArticleDetail/NODE07157994

Möhlmann, M. (2015). Collaborative consumption: determinants of satisfaction and the likelihood of using a sharing economy option again. Journal of Consumer Behaviour, 14(3), 193-207. https://doi.org/10.1002/cb.1512

Molz, J. G. (2013). Social networking technologies and the moral economy of alternative tourism: The case of couchsurfing. org. Annals of tourism research, 43, 210-230. https://doi.org/10.1016/j.annals.2013.08.001

Parigi, P., Santana, J. J., \& Cook, K. S. (2017). Online field experiments: studying social interactions in context. Social Psychology Quarterly, 80(1), 1-19. https://doi.org/10.1177/0190272516680842 
Park, M. H. (2016). Consumers' experiences of collaborative consumption based on sharing economy service. Journal of Consumer Studies, 27(27), 175-205. Retrieved from https://tinyurl.com/yycnf7al

Pauwels, M. C. (2015). Collaborative consumption in the United States. Revue LisaLisa e-Journal, 13(2). https://doi.org/10.1057/9781137473905 5

Pera, R., Viglia, G., \& Furlan, R. (2016). Who am I? How compelling self-storytelling builds digital personal reputation. Journal of Interactive Marketing, 35, 44-55. https://doi.org/10.1016/j.intmar.2015.11.002

Santoso, A. S., \& Erdaka, A. (2015). Customer loyalty in collaborative consumption model: empirical study of CRM for product-service system-based e-commerce in Indonesia. Procedia computer science, 72, 543-551. https://doi.org/10.1016/j.procs.2015.12.162

Seo, A., Jeong, J., \& Kim, Y. (2017). Cyber physical systems for user reliability measurements in a sharing economy environment. Sensors, 17(8). https://doi.org/10.3390/s17081868

Svetlana, K., \& YongIk, Y. (2016). Recommendation system for sharing economy based on multidimensional trust model. Multimedia Tools and Applications, 75(23), 15297-15310. https://doi.org/10.1007/s11042-014-2384-5

Teubner, T., \& Flath, C. M. (2015). The economics of multi-hop ride sharing. Business \& Information Systems Engineering, 575), 311-324. https://doi.org/10.1007/s12599-015-0396-y

ter Huurne, M., Ronteltap, A., Corten, R., \& Buskens, V. (2017). Antecedents of trust in the sharing economy: A systematic review. Journal of Consumer Behaviour, 16(6), 485-498. https://doi.org/10.1002/cb.1667

Weber, T. A. (2014). Intermediation in a sharing economy: insurance, moral hazard, and rent extraction. Journal of Management Information Systems, 31(3), 35-71. https://ssrn.com/abstract $=2439110$

Wu, J., Ma, P., \& Xie, K. L. (2017). In sharing economy we trust: the effects of host attributes on short-term rental purchases. International Journal of Contemporary Hospitality Management, 29(11), 2962-2976. https://doi.org/10.1108/IJCHM-08$\underline{2016-0480}$

Wu, J., Zeng, M., \& Xie, K. L. (2017). Chinese travelers' behavioral intentions toward room-sharing platforms: The influence of motivations, perceived trust, and past experience. International Journal of Contemporary Hospitality Management, 29(10), 2688-2707. https://doi.org/10.1108/IJCHM-08-2016-0481 
Yang, S., Song, Y., Chen, S.X., \& Xia, X. (2017). Why are customers loyal in sharingeconomy services? A relational benefits perspective. Journal of Services Marketing, 31(1), 48-62. https://doi.org/10.1108/JSM-01-2016-0042

Xie, K., \& Mao, Z. (2017). The impacts of quality and quantity attributes of Airbnb hosts on listing performance. International Journal of Contemporary Hospitality Management, 29(9), 2240-2260. https://doi.org/10.1108/IJCHM-07-2016-0345 